\title{
On Development of Sports Sociology from the Perspective of Functionalism
}

\author{
Dongsheng LV
}

Physical College, Jiujiang University, Jiujiang, 332005, China

\section{Keywords: Functionalism; Sports sociology; Development}

\begin{abstract}
Functionalism belongs to anthropology and sociology theories, and is also the most representative among them. Research and development of sports sociology are inseparable from functionalism. Study on modern functionalism theory and its application in sports sociology is significant for promoting the development of sports sociology. Based on this, this paper mainly discusses the development of sports sociology from the perspective of functionalism.
\end{abstract}

\section{Introduction}

Functionalism is another important theoretical school of modern western sociology following evolutionism and diffusionism, and it is also the research method commonly used by humanistic scientists and social scientists in their own area. Functionalism was the main theoretical basis at the early stage of sports sociology, and in the 1960s became the theory paradigm widely used in American sociological circle.

Functionalism belongs to anthropology and sociology theories. Also functionalism had effect on early study on western sports sociology, and became the most influential theoretical system. Especially from the 1930s to the1960s, structural-functional theory played a dominant role in North American sports sociology, which also had significant impact on research and development of sports sociology. However, in 1970s, western sports sociological scientists proposed huge doubts and criticism about functionalism, but soon later it was discovered that thinking method of research on sports sociology still followed the traditional functionalism. Since then, structural-functional theory mode had attracted much attention and research of many functionalist theorists, after which a lot of unknown mysteries were found in later research on sports sociology.

\section{Introduction to functionalism}

Functionalism emphasizes collaboration and cooperation of various parts of society to orderly achieves social needs, and it becomes a theoretical point of view which occupies an important position, also called "harmony theory" or "equilibrium theory". In sociology, "function" refers to that a social phenomenon, for the larger system, contains recognized and accepted reality conclusions. Various phenomena in the social system are interconnected, and any change of each part in the social system affects the whole system. One sentence in functionalism says that, a society is composed of a variety of different substances, to obtain a relatively stable and long-term structure of substance. However, the social structure contains various social systems, social organizations and a variety of behavior patterns, as well as distribution of various social roles. Each member in the social structure is significant in socialist theory because they have different roles in survival of overall society, so emphasis on the social system and regulate functioning of each role is necessary, conducive to virtuous cycle of society thus achieving balanced development. 


\section{Functions of sports from the perspective of functionalism}

\section{Maintain social system}

Same as with others social systems such as educational system, family system, economic system and political system, sports are the secondary system, but also play a key role in achieving social goals. Through participation in sports, people will have deeper understanding of social values, until they can achieve integration and regulation, digest these values based on their own understanding, to control their emotions, thinking and behavioral methods in order to make themselves cheerful and moral, especially allow minors to get a chance to exercise in society to help improve their sense of social responsibility and duty. This socialized exercise can better regulate common value system of the society, so as to create a good social order for better and faster development.

\section{Help achieve goals}

Sports attach particular importance to each goal of the society, and make them conform to laws and regulations. Sports are actually a process in which people internalize social significance and realize their own life goals and values, so that everyone knows, if we succeed we will certainly get a good return, but in order to achieve success, we have to redouble we efforts and sweat100times, and also ensure that the social system is not violated. Everyone knows that the fundamental purpose of sports is to get a good result, but it is also based on faire competition and no breach of rules. This requires us to use legitimate means to get the success, and absolutely cannot use despicable means to achieve our own goals. We require fair competition and refuse victory first. Through participation in sports, people can understand the significance of social progress designated through being committed to the society and unremitting efforts can bring realization of social progress.

\section{Promote social integration}

Interpersonal contact with each other forms the society, which is composed of a variety of people in all walks of life. The role of sports is to make people in all walks of life come together and form great cohesion force, thus reflecting the collective responsibility to safeguard emotions between all peoples, and ultimately achieve national unity, social stability and social integration.

\section{Better defense external threats}

Through participation in sports, we can learn a variety of life skills. In case of external threats, we will be able to calmly deal with them and improve our defense capabilities. We can also learn a lot of thinking, emotional and behavioral modes which adapt to the society. When the environment outside the system changes, we not only can protect ourselves, but also do not undermine internal order of the system, to achieve win-win.

\section{Study on development of sports sociology from the perspective of functionalism}

\section{Division and integration of sports sociology from the perspective of functionalism}

Sports sociology belongs to the social science, including many professional disciplines of university, such as sports economics, sports demography, sports industry, sports policies and regulations. Under the influence of functionalism and after a period of differential development, these disciplines will gradually tend to be integrated and comprehensive; affected by functionalism, many major and leading topics attract and converge various disciplines together, and then new development direction of disciplines appears. Social sports science and other cognate disciplines will steadily achieve fusion under functionalism. Interdisciplinary research of sports sociology based on functionalism becomes commonplace. Branches of social sports science will gradually realize the trend from division to integration under the guidance of functionalism. 


\section{Gradual localization of discipline construction of sports sociology}

Undoubtedly, the concept of localization refers to that discipline development should be in line with our national situation. In the future, localization will guide the development trend of sports sociology, which is a good opportunity for developing China. China's traditional sports have a long history, also highly recognized by our people, so we should strive to develop our traditional sports, and make them take their own proper place in the world of sports. This has two historical significances here. On one hand, our traditional sports can obtain better inheritance and development, and will not be eliminated by western sports; on the other hand, it can enrich sports culture in the world, making worldwide sports culture more prosperous.

\section{Globalization, intellectualization and network development}

As far as right now, there are more and more interdisciplinary researches of sports sociology. In the early 1980s, sports sociology in China first sprung up, as well as sports management science, economics, law, introduction, philosophy, ethics and other disciplines. Various disciplines affected and permeated each other; so the boundary of sports sociology was unclear, and there was no well-defined concept. After the 1990s, it was more common that sports sociology overlapped with other disciplines, such as, sports management science, philosophy, culture, etc. especially it combined with sports economics. So the discussion of Chinese sports sociology phenomena is very representative. On the other hand, scholars in other fields that previously did not pay attention to research of sports sociology have turned to this research. They brought traces of the original discipline, resulting in abound interdisciplinary researches. This is also the basic reason for unclear boundary of sports sociology research. In the future, the important feature of sports sociology research is collective research and horizontal integration. I hope with new research methods and angles, integration of sports science branches will be soon realized.

\section{Sports sociology has great development potential and discipline construction admits of no delay}

According to China's national conditions, sports sociology research has large development space and good opportunities, but research efforts on various branches of sports sociology at this stage still need to be strengthened, including: sports economics, political science, industry science, policy science, law and so on. Although research on various branches of sports sociology proceeds rapidly, but because development of various branches is not mature, they cannot yet become mature disciplines. Therefore, it is a long way to go to take advantage of characteristics of various branches to enrich and develop sports sociology. On the other hand, we should attract staff from integrated humanities to work together on the effective study on some major issues about social sports, such as study on sports health knowledge, comprehensively promoting health science knowledge, sustainable development of sports industry directly related to China's economy, emphasizing and strengthening timeliness of major sports sociology issues, Olympic spirit and related culture popularization and promotion, inheriting and carrying forward the national tradition, and multinational cooperation and comparative studies of worldwide sports science. At the same time we must combine political, cultural, economic characteristics in China, not blindly copy from others without thinking.

\section{Theoretical evaluation of sports sociology development from the perspective of functionalism}

According to the basic theoretical thought and theoretical system of functionalism, sports are part of the social system, which have a very important role in maintaining social stability. This theoretical perspective focuses on the role of sports in promoting social development, also the special role in social harmonious progress, self-improvement of people, and national political decision-making, etc. However, studies on sports sociology issues under the influence of functionalism have limitations. Some scholars have suggested that functionalism just focuses on the positive effects of sports, but ignored their negative impact on some people; some conflicts of interest in sports can naturally get integrated at equilibrium state, but functionalism in reality is different from that in ideal conditions; 
however, as a social structure, functionalism, in fact, is more conducive to rich and powerful groups, which will produce social instability and fuel instability factors.

The biggest deficiency of sports science development is also generated under the influence of functionalism theory, because functionalism theory supports sports are the same for everyone and it pays too much attention to the positive role of sports and ignores their negative impact. For example, gender differences not able to be changed exist in sports, which will bring unfair treatment for women in society; permanent class differences also exist in sports, which will bring unfair treatment for the poor. Sports might change their values and principles in life of some people, but sometimes also may dampen one's enthusiasm. So, some powerful or influential people are more inclined to use structural-functional theory for analyzing and solving problems, because they pay more attention to social stability and justice.

Structural-functional theory has a significant feature. That is the focus on the static and conservative analysis on social system. This feature is not necessary for structural- functional theory, but also gradually becomes the basic knowledge of the theory. For conservatives or people who want social stability, they favor structural-functional theory. Restructuring and transformation of society is reflected in functionalism all the time, but they believe social transformation follows the basic mode of human evolution, and the cause of social chaos and anomalies is constantly change of society, resulting in maladaptation of the old social system. So functionalism occupies a prominent position in national development, always influencing the transformation of social systems including sports. They believe that transformation of sports requires a period of evolution process, so as to always give play to various functions of sports and create a more harmonious and stable society.

\section{Conclusions}

In summary, the purpose of functionalism is to help society, communities, organizations and groups, and it focuses on how society stably operates, and also studies how individuals participate in sports, and how they make due contributions to the society in sports. In contemporary Chinese society, we need functionalism to make correct guidance, study how to maintain social stability, regulate personal behaviors, and ultimately achieve the harmonious unity of national policy, economy and culture with the help of national sports. But functionalism also has some disadvantages, which requires us to actively think about its application. For example, it only focuses on the consistency of social needs, but ignores the interest of different sports groups, and disregards social diversity. These deficiencies to some extent limit the development of Chinese sports in the early stages of social transformation, and also prevent us from deep investigation and exploration.

\section{References}

[1] National Policy and Regulation Department, American Sports Sociology. Compilation of Soft Science Research on Sports, 2013: 40-49.

[2] Li Bo, Zheng Xiuli. American Competitive Sports from the Perspective of Sociological Theory. Journal Shenyang Institute of Physical Education, 2009, 28 (5): 26-29.

[3] Lu Jifeng. Effect and Enlightenment of Functionalism on Education Research. Daizong Journal, 2011, 15 (3): 96-97.

[4] Zhang Xinping. Sports Sociology Traceability and Its Enlightenment on the Development of Chinese Sports Sociology in New Century. Journal of Shandong Institute of Physical Education, 2013, (12): 143-144.

[5] Lu Jifeng. Effect and Enlightenment of Structural-.Functional Theory on Education Research. Daizong Journal in Taian College of Education, 2011, (03). 